\title{
Average consensus problems in networks of agents with delayed communications
}

\author{
Pierre-Alexandre Bliman ${ }^{\mathrm{a}, 1}$ Giancarlo Ferrari-Trecate ${ }^{\mathrm{a}}$ \\ ${ }^{\mathrm{a}}$ INRIA, Domaine de Voluceau \\ Rocquencourt - B.P.105, 78153, Le Chesnay Cedex, France \\ e-mail: $\{$ Pierre-Alexandre.Bliman, Giancarlo.Ferrari-Trecate\}@inria.fr
}

\begin{abstract}
The present paper is devoted to the study of average consensus problems for undirected networks of dynamic agents having communication delays. The accent is put here on the study of the time-delays influence: both constant and time-varying delays are considered, as well as uniform and non uniform repartitions of the delays in the network. The main results provide sufficient conditions (also necessary in most cases) for existence of average consensus under bounded, but otherwise unknown, communication delays. Simulations are provided that show adequation with these results.
\end{abstract}

\section{Introduction}

In the last few years, the study of multi-agent systems has received a major attention within the control community. Driving applications include unmanned aerial vehicles, satellite clusters, automated highways and mobile robots. In all cases the aim is to control a group of agents connected through a wireless network. More precisely, rather than stabilizing the movement of each agent around a given set point, the goal is to understand how to make the agents coordinate and self-organize in moving formations. This problem becomes even more challenging under partial communication protocols, i.e. when each agent exchanges information only with few others.

Many works in the literature focused on conditions for guaranteeing that the agents asymptotically reach a consensus, i.e. they agree upon a common value of a quantity of interest [11], [18], [19], [16], [17], [4]. As an example, in a network of moving vehicles a form of consensus is represented by alignment, that

$\overline{1}$ Corresponding author. Tel: +33 1396355 68, Fax:+33 139635786 . 
happens when all vehicles asymptotically move with the same velocity. In the aforementioned papers, consensus problems have been studied under a variety of assumptions on the network topology (fixed/switching), the communication protocol (bidirectional or not), additional performance requirements (e.g. collision avoidance, obstacle avoidance, cohesion), and the control scheme adopted (also termed consensus protocol). So far, just few works considered consensus problems when communication is affected by time-delays. Some results for discrete-time agent models are given in [5] and [1]. Two different consensus protocols for continuous-time agent dynamics have been investigated in [14] and [16]. More specifically, assuming that agents behave like integrators and that communication delays are constant in time and uniform (i.e. they have the same value in all channels), an analysis of the maximal delay that can be tolerated without compromising consensus has been performed in [14] and [16]. In particular, the protocol adopted in [16] is capable to guarantee average consensus (i.e. the state of each agent converges, asymptotically, to the average of the initial agent states rather than to an arbitrary constant) and the authors provide an explicit formula for the largest transmission delay.

In the present work we generalize the results of [16] in various ways. First, we consider uniform and unknown time-varying delays and provide upper bounds to the maximal delay that does not prevent from achieving average consensus. Second, we derive similar conditions for networks affected by non uniform, constant or time-varying delays. In the case of non uniform and constant delays, we also show that if the communication delay between two agents is equal to zero, then average consensus may achieved irrespectively of the magnitude of all others delays.

The network of agents is modeled in the framework of Partial difference Equations (PdEs) introduced in [2] and used in [4] analyzing the property of various linear and nonlinear consensus protocols. PdEs are models that mimic Partial Differential Equations (PDEs) and provide a mathematical description of the agents network where "spatial" interactions (due to the network structure) and "temporal" ones are kept separated and described by operators acting either on space or time. Section 2 provides an introduction to PdEs. The main results are presented through Sections 3-6 and three simulation experiments are discussed in Section 7. The upper bounds to the maximal tolerable delay depend on some eigenvalues of suitably defined operators. Although their numerical computation is easy for a given network, in Section 7.1 we provide their explicit form as a function of the network size for fully connected and loop-shaped network.

\section{Tools for functions on graphs}

The communication network is modeled through an undirected weighted graph $G$ defined by a set $\mathcal{N}=\{1,2, \ldots, N\}$ nodes and a set $\mathcal{E} \subset \mathcal{N} \times \mathcal{N}$ of edges. 


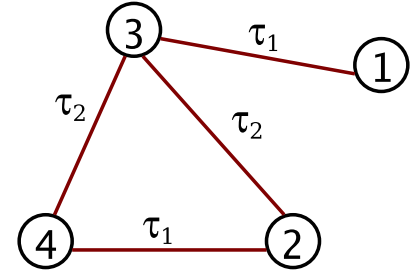

(a) Graph $G$.

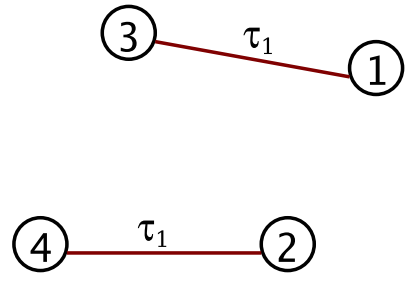

(b) Subgraph $G_{1}$.

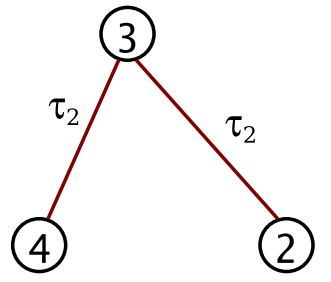

(c) Subgraph $G_{2}$.

Fig. 1. A graph and the subgraphs associated to delays $\tau_{1}$ and $\tau_{2}$

Each node represents an agent and an edge $(x, y)$ means that the agents $x$ and $y$ share the information about their states. Agents linked by an arc are called neighbors. The neighboring relation is denoted with $x \sim y$ and we assume that $x \sim x$ always holds. Two nodes $x$ and $y$ are connected by a path if there is a finite sequence $x_{0}=x, x_{1}, \ldots, x_{n}=y$ such that $x_{i-1} \sim x_{i}$. The graph $G$ is connected when each pair of nodes $(x, y) \in G \times G$ is connected by a path and complete if $\mathcal{E}=G \times G$.

Weights on the communication links are defined by a function $\omega: \mathcal{N} \times \mathcal{N} \mapsto \mathbb{R}^{+}$ with the properties

$$
\begin{aligned}
& \omega(x, y)=\omega(y, x) \\
& \omega(x, y)>0 \Leftrightarrow x \sim y
\end{aligned}
$$

Time-varying delays in communications, are elements of the set

$$
\mathcal{D}=\left\{\tau_{i}(\cdot): i \in \mathcal{I}\right\}, \quad \mathcal{I}=\{1, \ldots, r\}, \quad r \leq \frac{N(N-1)}{2}
$$

where $\tau_{i}: \mathbb{R}_{+} \mapsto \mathbb{R}_{+}$are piecewise continuous functions. A delay is associated to each edge through the onto function $T: \mathcal{E} \mapsto \mathcal{D}$ verifying $T(x, y)=T(y, x)$. The last equality amounts to consider delays that are symmetric, i.e. the lags in transmission from $x$ to $y$ and from $y$ to $x$ do coincide. This also motivates the bound $r \leq \frac{N(N-1)}{2}$ in $(2)$.

Agents linked with the same delay $\tau_{i}(\cdot)$, define a subgraph $G_{i}=\left(\mathcal{N}, T^{-1}\left(\tau_{i}\right)\right)$ with associated weights

$$
\omega_{i}(x, y)= \begin{cases}\omega(x, y) & \text { if }(x, y) \in T^{-1}\left(\tau_{i}\right) \\ 0 & \text { otherwise }\end{cases}
$$

An example is reported in Figure 1. We highlight that the subgraphs $G_{i}$ may be disconnected even if $G$ is connected. Moreover, as shown in Figure 1(c), some nodes can be isolated.

We consider now vector functions $f: \mathcal{N} \mapsto \mathbb{R}^{d}$ defined over a graph $G$. For instance, $f(x)$ may represent the position or the velocity of the agent $x$ at a 
fixed time-instant. Following [2], the partial derivative of $f$ is defined as

$$
\partial_{y} f(x) \doteq f(y)-f(x)
$$

and enjoys the following basic properties:

$$
\begin{aligned}
& \partial_{y} f(x)=-\partial_{x} f(y) \\
& \partial_{x} f(x)=0 \\
& \partial_{y}^{2} f(x)=\partial_{y} f(y)-\partial_{y} f(x)=-\partial_{y} f(x)
\end{aligned}
$$

The integral and average of $f$ are defined, respectively, as

$$
\int_{G} f d x \doteq \sum_{x \in \mathcal{N}} f(x), \quad\langle f\rangle \doteq \frac{1}{N} \int_{G} f d x .
$$

Note that, in (6), " $d x$ " just indicates the integration variable. The Laplacian of $f$ is given by

$$
\Delta f(x) \doteq-\sum_{y \sim x} \omega(x, y) \partial_{y}^{2} f(x)=+\sum_{y \sim x} \omega(x, y) \partial_{y} f(x) .
$$

where the last identity follows from (5c). In an equivalent way, the Laplacian can be written as

$$
\Delta f(x)=\int_{G} \omega(x, y) \partial_{y} f(x) d y
$$

The Laplacian operator associated to a subgraph $G_{i}$ is

$$
\Delta_{i} f(x) \doteq \int_{G} \omega_{i}(x, y) \partial_{y} f(x) d y
$$

Since the sets of edges $\left\{T^{-1}\left(\tau_{i}\right)\right\}_{i \in \mathcal{I}}$ are a partition of $\mathcal{E}$, it is immediate to verify that

$$
\omega(x, y)=\sum_{i \in \mathcal{I}} \omega_{i}(x, y) \quad \text { and } \quad \Delta f=\sum_{i \in \mathcal{I}} \Delta_{i} f
$$

In the sequel we summarize the main properties of the Laplacian operator stated in [2]. The driving idea is to mimic functional analysis tools for studying the classic Laplacian defined on Sobolev spaces, (see [2] and [4] for further details).

We denote with $L^{2}\left(G \mid \mathbb{R}^{d}\right)$ the Hilbert space composed by all functions $f$ : $\mathcal{N} \mapsto \mathbb{R}^{d}$ equipped with the scalar product and the norm

$$
(f, g)_{L^{2}}=\int_{G} f \cdot g, \quad\|f\|_{L^{2}}^{2}=\int_{G}\|f\|^{2}
$$

where $\cdot$ and $\|\cdot\|$ represent the scalar product and the euclidean norm on $\mathbb{R}^{d}$, respectively. Let $H^{1}\left(G \mid \mathbb{R}^{d}\right)$ be the space collecting all functions in $L^{2}\left(G \mid \mathbb{R}^{d}\right)$ with zero average. We will use the shorthand notation $L^{2}$ and $H^{1}$ when there 
is no ambiguity on the underlying domain and range of the functions. If $G$ is connected, $H^{1}$ is an Hilbert space [2] endowed with scalar product

$$
(f, g)_{H^{1}}=\int_{G} \int_{G} \omega(x, y) \partial_{y} f(x) \cdot \partial_{y} g(x) d x d y
$$

Apparently, $H_{\perp}^{1}$ is the space of constant functions on $G$ and $\operatorname{dim}\left(H_{\perp}^{1}\right)=d$. Moreover, the decomposition $L^{2}=H^{1} \oplus H_{\perp}^{1}$ is direct. The $L^{2}$ orthogonal projection operators on $H^{1}$ and $H_{\perp}^{1}$ will be denoted as $P_{H^{1}}$ and $P_{H_{\perp}^{1}}$, respectively.

The eigenstructure of the Laplacian is completely characterized by the next Theorem, proved in [2].

Theorem 1 Let $G$ be a connected graph. Then,

(1) the operator $\Delta: H^{1} \mapsto H^{1}$ is symmetric, it has $(N-1) d$ strictly negative eigenvalues $^{2}$ and the corresponding eigenfunctions form a basis for $H^{1}$;

(2) for $f \in L^{2}, \Delta f=0$ if and only if $f \in H_{\perp}^{1}$.

Theorem 1 highlights that the Laplacian is invertible on the subspace $H^{1}$. Note that when $\Delta$ is defined on $L^{2}$, it has $N d$ eigenvalues. In particular, in view of the decomposition $L^{2}=H^{1} \oplus H_{\perp}^{1},(N-1) d$ eigenvalues are those considered in point (1) of Theorem 1 and the remaining $d$ are zeros (this property follows directly from point (2) of Theorem 1).

The next theorem characterizes the eigenvalues of the operators $\Delta_{i}$.

Theorem 2 The operators $\Delta_{i}: H^{1} \mapsto H^{1}, i \in \mathcal{I}$, are symmetric and negativesemidefinite.

Proof: As in the proof of [2, Lemma 3.1], by direct calculation one has that $\forall u, v \in L^{2}$ it holds $\left(\Delta_{i} u, v\right)_{L^{2}}=-a_{i}(u, v)$ where $a$ is the symmetric bilinear form given by

$$
a_{i}(u, v) \doteq \frac{1}{2} \int_{G_{i}} \int_{G_{i}} \omega_{i}(x, y) \partial_{y} u(x) \cdot \partial_{y} v(x) d x d y
$$

Then, $(\Delta u, v)_{L^{2}}=-a(u, v)=-a(v, u)=(u, \Delta v)_{L^{2}}$ that proves the symmetry of $\Delta$. For proving that $\Delta_{i}$ is negative semidefinite, it is enough to show that $\left(-\Delta_{i} u, u\right)_{L^{2}} \geq 0, \forall u \in L^{2}$. One has

$$
\left(-\Delta_{i} u, u\right)_{L^{2}}=a_{i}(u, u)=\frac{1}{2} \int_{G_{i}} \int_{G_{i}} \omega_{i}(x, y)\left\|\partial_{y} u(x)\right\|^{2} d x d y
$$

where the last term is non negative in view of (3) and (1b).

We stress that all the spaces so far introduced are finite dimensional. This can be seen by noting that the lifting operator $\mathcal{L}: L^{2}\left(G \mid \mathbb{R}^{d}\right) \mapsto \mathbb{R}^{N d}$ defined as

$$
\mathcal{L}(f) \doteq\left[f(1)^{T} \cdots f(N)^{T}\right]^{T}
$$

$\overline{2}$ Such eigenvalues will be termed "the eigenvalues of $\Delta$ on $H^{1}$ ". 
is an isometry (i.e. bijective and $\left.\|f\|_{L^{2}}=\|\mathcal{L}(f)\|\right)$ so showing that $L^{2}$ is isomorphic to $\mathbb{R}^{N d}$. Roughly speaking, this means that all concepts introduced in the present section could be re-written in terms of vector and matrices over $\mathbb{R}^{N d}$.

Definition 1 Consider the linear operator $A: L^{2}\left(G \mid \mathbb{R}^{d}\right) \mapsto L^{2}\left(G \mid \mathbb{R}^{d}\right)$. Its matrix representation is the unique matrix $\mathcal{M}(A) \in \mathbb{R}^{N d \times N d}$ that verifies $\mathcal{L}(A f)=\mathcal{M}(A) \mathcal{L}(f), \forall f \in L^{2}\left(G \mid \mathbb{R}^{d}\right)$.

The matrix representation of an operator can be used, for instance, for computing the eigenvalues of $A$ since they coincide with the eigenvalues of $\mathcal{M}(A)$, up to their multiplicity. The operator $\Delta$ is strongly related to the Laplacian matrix of the graph $G$, defined next (see also [3]). In the sequel, the $x$-th row and the $(x, y)$ element of a matrix $B$ will be denoted with $(B)_{x}$ and $(B)_{x, y}$, respectively.

Definition 2 For a graph $G$, the adjacency matrix $A(G)$ is an $N \times N$ matrix with entries

$$
(A(G))_{x, y} \doteq \begin{cases}\omega(x, y) & \text { if } x \sim y \text { and } x \neq y \\ 0 & \text { otherwise }\end{cases}
$$

The valency matrix $V(G)$ is an $N \times N$ diagonal matrix with entries $(V(G))_{x, x} \doteq$ $\sum_{y \sim x} \omega(x, y)$ and the Laplacian matrix is $L(G) \doteq A(G)-V(G)$.

It is easy to verify that $\mathcal{L}(\Delta f(x))=\left(L(G) \otimes I_{d}\right) \mathcal{L}(f)$, where $\otimes$ is the Kronecker product and $I_{d}$ the identity matrix of order $d$ Then, $\mathcal{M}(\Delta)=L(G) \otimes I_{d}$.

\section{Delayed multi-agent models and PdEs}

Let $v(x, t) \in \mathbb{R}^{d}$ and $u(x, t) \in \mathbb{R}^{d}, x \in \mathcal{N}, t \in \mathbb{R}^{+}$denote the state and the control input of agent $x$ at time $t$, respectively. When each agent behaves as an integrator, the collective dynamics is described by the equation $\dot{v}(x, t)=$ $u(x, t)$, where the dot operator indicates the time-derivative. In this paper we consider delayed Laplacian protocols of the type $u=\sum_{i \in \mathcal{I}} \Delta_{i} v\left(x, t-\tau_{i}(t)\right)$ yielding the collective dynamics

$$
\dot{v}=\sum_{i \in \mathcal{I}} \Delta_{i} v\left(x, t-\tau_{i}(t)\right)
$$

Formula (17) defines a time-delay Partial difference Equation (PdE) (see [4] for a general definition of PdEs) whose solution depends on the initial conditions. As for linear time-delay systems, if all delays are bounded by a constant $\bar{\tau}$, the initial condition may be given in form of a function $\tilde{v}(x, t) \in \mathbb{R}^{d}, x \in \mathcal{N}$, $t \in[-\bar{\tau}, 0]$, continuous in $t$.

As shown in [4], PdEs can be always recast into Ordinary Differential Equations by using the lifting operator (15). Then, it is not surprising that linear time-delay PdEs inherit all the properties of linear time-delay systems. As an 
example, if all delays are constant in time, the characteristic equation associated to $(17)$, is

$$
E(s) \doteq s I-\sum_{i \in \mathcal{I}} e^{-s \tau_{i}} \Delta_{i}=0, \quad s \in \mathbb{C}
$$

where $I$ is the identity operator on $L^{2}$. Then, many properties of the network of agents can be characterized in terms of the poles of (17), i.e. the roots of (18). We outline that if the delays are constant, model (17) coincides with the network dynamics considered in Section 10 of [16].

The main goal of the present work is to investigate when (17) guarantees average consensus.

Definition 3 The network dynamics achieves average consensus if $v \rightarrow\langle v(\cdot, 0)\rangle$ as $t \rightarrow+\infty$.

In absence of delays, $u$ results in the Laplacian protocol, and the PdE (17) reduces to the heat equation

$$
\dot{v}=\Delta v \quad v(\cdot, 0)=\tilde{v} \in L^{2}
$$

The consensus properties of Laplacian protocols have been analyzed in various works. In particular, A. Jadbabaie et al. [11] proved that the Laplacian protocol is able to guarantee average consensus under various assumption on the network topology. A formal analysis of the PdE (19) has been carried out in [4], where it has been also shown that the Laplacian protocol can guarantee consensus even when the agent dynamics are perturbed by exponentially decreasing errors and/or an agent acts as the leader of the group.

In order to highlight the rationale we will use for analyzing the PdE (17), let us summarize the main results of [4] for the collective dynamics (19). Decomposing the velocities as $v=v_{1}+\bar{v}, v_{1} \in H_{1}, \bar{v}=\langle v(\cdot, t)\rangle \in H_{\perp}^{1}$, one can show, through a simple variational technique, that the velocity components fulfill the dynamics

$$
\begin{aligned}
\dot{\bar{v}} & =0 \\
\dot{v}_{1} & =\Delta v_{1}
\end{aligned}
$$

thus proving that the spaces $H_{1}$ and $H_{\perp}^{1}$ are positively invariant for (19). In particular, equation (20a) highlights that the average velocity of the agents is constant in time. Then an exponentially stable average consensus is achieved if the origin of (20b) is exponentially stable, a fact that can be easily shown by exploiting the characterization of the eigenvalues of $\Delta$ on $H^{1}$ given in Theorem 1. In [4] it is also shown that average consensus can be intuitively expected on the basis of the physical analogy between (19) and the classic heat equation.

For the delayed model (17), we will adopt a similar argument. The next Lemma provides the dynamics of the $v_{1}$ and $\bar{v}$ components. 
Lemma 1 The function $v$ is solutions to the PdEs (17) if and only if $v_{1}$ and $\bar{v}$, are solutions to the PdEs

$$
\Sigma_{1}: \dot{v}_{1}=\sum_{i \in \mathcal{I}} \Delta_{i} v_{1}\left(x, t-\tau_{i}(t)\right), \quad \bar{\Sigma}: \dot{\bar{v}}=0
$$

equipped with the initial conditions $v_{1}(\cdot, t)=P_{H^{1}} \tilde{v}(\cdot, t), \bar{v}(t)=P_{H_{\perp}^{1}} \tilde{v}(\cdot, t)$ for $t \in[-\bar{\tau}, 0]$.

Proof: To prove the result, we use a variational argument by testing each side of (17) against all $c \in H_{\perp}^{1}$. This means that we take the integrals

$$
\int_{G} c \cdot \dot{v} d x=\int_{G} c \cdot \sum_{i \in \mathcal{I}} \Delta_{i} v\left(x, t-\tau_{i}(t)\right) d x
$$

By using (9), the right side of (22) can be written as $\sum_{i \in \mathcal{I}} S_{i}$, where

$$
S_{i}=\int_{G} c \cdot \int_{G} \omega_{i}(x, y) \partial_{y} v\left(x, t-\tau_{i}(t)\right) d y d x
$$

From (1a) and (5a), the functions $g_{i}(x, y)=\omega_{i}(x, y) \partial_{y} v\left(x, t-\tau_{i}(t)\right)$ are antisymmetric, i.e. $g_{i}(x, y)=-g_{i}(y, x)$. Then, each integral $S_{i}$ can be expanded into sums containing only terms of the type $c \cdot\left(g_{i}(x, y)+g_{i}(y, x)\right)$ that are all identically equal to zero. The fact that $\int_{G} c \cdot \dot{v} d x=0, \forall c \in H_{\perp}^{1}$ corresponds to the condition $P_{H_{\perp}^{1}} \dot{v}=0$, or, equivalently, to $\dot{\bar{v}}=0$, thus obtaining the dynamics $\bar{\Sigma}$. From (17) we have

$$
\dot{v}_{1}+\dot{\bar{v}}=\sum_{i \in \mathcal{I}} \Delta_{i} v_{1}\left(x, t-\tau_{i}(t)\right)+\sum_{i \in \mathcal{I}} \Delta_{i} \bar{v}\left(x, t-\tau_{i}(t)\right)
$$

and the dynamics $\Sigma_{1}$ follows from $\dot{\bar{v}}=0$ and $\Delta_{i} \bar{v}=0$.

Lemma 1 shows that the spaces $H^{1}$ and $H_{\perp}^{1}$ are positively invariant for the $\operatorname{PdE}(17)$. Moreover, as for (19), the average state $\bar{v}$ is constant in time and equal to $\langle\tilde{v}(\cdot, 0)\rangle$. Then, the problem of checking average consensus is reduced to the problem of proving that $v_{1} \rightarrow 0$ as $t \rightarrow \infty$. We also say that average consensus is globally exponentially or asymptotically stable if the zero solution to $\Sigma_{1}$ enjoys the same property, i.e. it is exponentially or asymptotically stable for all initial conditions $\tilde{v}(\cdot, t) \in H^{1}, t \in[-\bar{\tau}, 0]$, continuous in $t$.

For subsequent use, we introduce the operator norm $\|\Delta\| \doteq \max _{u \in H^{1}} \frac{(u, \Delta u)}{(u, u)}=$ $\left|\lambda_{\text {min }}\right|$ where $\lambda_{\text {min }}$ is the minimal eigenvalue of the Laplacian on $H^{1}$. Similarly, by recalling that $\Delta$ is invertible on $H^{1}$, one has $\left\|\Delta^{-1}\right\|^{-1}=\left|\lambda_{\max }\right|$.

\section{The case of uniform delays}

In this section, we analyze the stability properties of the dynamics $\Sigma_{1}$ when the delay is uniform in the network, i.e. when $\mathcal{I}$ is a singleton. We start with 
the simpler case of time-invariant delays, considered also in [16]. The results of the next Theorem coincide with those of Theorem 10 in [16], but are proved through a different argument, i.e. the diagonalization of the Laplacian operator on $H^{1}$.

Theorem 3 (Constant delay) The zero function is a globally exponentially stable solution to the PdE

$$
\dot{v}_{1}(x, t)=\Delta v_{1}(x, t-\tau), \quad v_{1} \in H^{1}
$$

for all possible $\tau \leq \bar{\tau}$, if and only if

$$
\bar{\tau}<\frac{\pi}{2\|\Delta\|}
$$

Proof: In view of Theorem 1, the Laplacian can be diagonalized on $H^{1}$. Let $\left\{\psi_{i}\right\}_{i=1}^{(N-1) d}$ be an orthonormal set of eigenfunctions of $\Delta$ forming a basis for $H^{1}$ and associated to the eigenvalues $\left\{\lambda_{i}\right\}_{i=1}^{(N-1) d}$. Then $v_{1}(x, t)=\sum_{i=1}^{(N-1) d} \alpha_{i}(t) \psi_{i}(x)$ for suitable functions $\alpha_{i}: \mathbb{R}^{+} \mapsto \mathbb{R}$. By testing each side of (25) against $\psi_{j}$ we form the integrals

$$
\int_{G}\left(\sum_{i=1}^{(N-1) d} \dot{\alpha}_{i}(t) \psi_{i}(x)\right) \cdot \psi_{j}(x) d x=\int_{G}\left(\sum_{i=1}^{(N-1) d} \alpha_{i}(t-\tau) \Delta \psi_{i}(x)\right) \cdot \psi_{j}(x) d x
$$

By Theorem 1, formula (27) reduces to

$$
\dot{\alpha}_{j}(t)=\lambda_{j} \alpha_{j}(t-\tau)
$$

System (28) is a first-order linear time-delay system. Since $\lambda_{j}<0$, according to $\left[9\right.$, Theorem A.5], system (28) is exponentially stable if and only if $\tau<\frac{\pi}{2\left|\lambda_{j}\right|}$. Then, the PdE (25) is exponentially stable if and only if all systems (28), for $j=1, \ldots,(N-1) d$ are exponentially stable, i.e. if (26) holds.

Remark 1 For $h \geq 0$, it may be of interest to quantify the largest delay $\bar{\tau}_{h}$ for which an exponential decay rate $h$ is guaranteed for the solutions to (25). By using the diagonalization procedure in the proof of Theorem 3, and noting that systems (28) are asymptotically stable for $\tau=0$, the quantity $\bar{\tau}_{h}$ is the smallest $\tau>0$ for which there exists $\omega \in \mathbb{R}$ such that

$$
-h+j \omega-e^{\tau(h-j \omega)}\|\Delta\|=0 .
$$

Using the fact that if $\omega$ verifies (29) also $-\omega$ verifies (29), one obtains $\omega=$ $\sqrt{\|\Delta\|^{2} e^{2 h \tau}-h^{2}}$. Furthermore, summing up the real and complex parts of equation (29) yields

$$
\bar{\tau}_{h} \doteq \min \left\{\tau \geq 0:\|\Delta\| e^{h \tau} \cos \left(\tau \sqrt{\|\Delta\|^{2} e^{2 h \tau}-h^{2}}\right)=h\right\}
$$


Note that the map $h \mapsto \bar{\tau}_{h}$ is decreasing, with $\bar{\tau}_{0}=\pi / 2\|\Delta\|$, and $\bar{\tau}_{\|\Delta\|}=0$.

We consider now the case of a single time-varying delay.

Theorem 4 (Time-varying delay) The zero solution is a globally exponentially stable solution to the PdE

$$
\dot{v}_{1}(x, t)=\Delta v_{1}(x, t-\tau(t)), \quad v_{1}(\cdot, t) \in H^{1}
$$

for all piecewise continuous delays $\tau(t)$ verifying $0 \leq \tau(t) \leq \bar{\tau}$, if and only if

$$
\bar{\tau}<\frac{3}{2\|\Delta\|} .
$$

Proof: As in the proof of Theorem 3, diagonalization of the Laplacian on $H^{1}$ leads to the study of the first-order systems $\dot{\alpha}_{i}=\lambda_{i} \alpha_{i}(t-\tau(t))$, for any eigenvalue $\lambda_{i}$ of $\Delta$ on $H^{1}$.

The conclusion is then deduced from a classical result initially published in [15] and [20], (see also [9, p. 164] and the references therein).

If the nominal collective model is the $\operatorname{PdE}(19)$, Theorems 3 and 4 characterize the robustness of average consensus with respect to different delay models. In particular, the bounds given in Theorems (26) and (31) do not depend upon the precise structure of the communication network but only upon the magnitude of $\|\Delta\|$. In other words, by interpreting $G$ as the "spatial" domain of the PdEs (25) and (30), bounds (26) and (31) relate the maximal tolerated delays to a spatial feature. Explicit formulas for $\|\Delta\|$ in the case of complete and loop-shaped networks are given in Sections 7.1.1 and 7.1.2, respectively. Other results linking the graph structure with the eigenvalues of the Laplacian operator can be found in [7], [6] and [13].

We also outline that the constant in (31) is smaller than the corresponding one in (26), the greater conservativity arising from the time-varying nature of the delay. However, the bound (31) is the best possible one since the corresponding stability condition is necessary and sufficient.

\section{The case of non-uniform delays}

In this Section, we generalize the results of Section 4 to the case where the delays do not take a common value in the whole network. Let us consider first the case of constant delays. The next Theorem provides a robust stability result for all possible delays $\tau_{i}$ within the interval $[0, \bar{\tau}]$. Quite remarkably, the bound (26) still gives a necessary and sufficient condition for stability.

Theorem 5 (Constant delays) The zero solution is a globally exponentially 
stable solution to the equation

$$
\dot{v}_{1}(x, t)=\sum_{i \in \mathcal{I}} \Delta_{i} v_{1}\left(x, t-\tau_{i}\right), \quad v_{1}(\cdot, t) \in H^{1}
$$

for all possible $\tau_{i} \leq \bar{\tau}, i \in \mathcal{I}$, if and only if (26) holds.

Proof: By considering the case $\tau_{i}=\bar{\tau}, i \in \mathcal{I}$, Theorem 3 shows that the upper bound to the tolerated delay cannot be larger than $\pi / 2\|\Delta\|$.

We prove by contradiction that (26) implies asymptotic stability. Assume that (32) is not asymptotically stable. For zero delays, the PdE (32) reduces to PdE (20b) whose global exponential stability has been proved in [4]. By continuity of the poles of (32) with respect to the delays, there exists a choice of the $\tau_{i} \in[0, \bar{\tau}], i \in \mathcal{I}$, for which the $\operatorname{PdE}(32)$ has a purely imaginary pole $j \omega, \omega \in$ $\mathbb{R} \backslash\{0\}$. In other words, there exists a nonzero eigenfunction $u \in H^{1}\left(G \mid \mathbb{C}^{d}\right) \backslash\{0\}$ such that

$$
\left(j \omega I-\sum_{i \in \mathcal{I}} \Delta_{i} e^{-j \tau_{i} \omega}\right) u=0,
$$

where $I$ is the identity on $H^{1}$. This implies that

$$
j \omega+\sum_{i \in \mathcal{I}} \alpha_{i} e^{-j \tau_{i} \omega}=0
$$

where, by denoting with $u^{*}$ the complex conjugate of $u$, one has

$$
\alpha_{i} \doteq-\frac{\int_{G} u^{*} \cdot \Delta_{i} u}{\int_{G} u^{*} \cdot u}, i \in \mathcal{I}
$$

Notice that, by Theorem 2 , the operators $\Delta_{i}$ are symmetric, negative semidefinite on $H^{1}$. Thus, one has $\alpha_{i} \geq 0$ for all $i \in \mathcal{I}$. On the other hand,

$$
\sum_{i \in \mathcal{I}} \alpha_{i} \leq\|\Delta\|
$$

Considering the real and imaginary parts of (33), we deduce that

$$
\begin{gathered}
\sum_{i \in \mathcal{I}} \alpha_{i} \cos \omega \tau_{i}=0, \\
\omega-\sum_{i \in \mathcal{I}} \alpha_{i} \sin \omega \tau_{i}=0 .
\end{gathered}
$$

From (34) and (35b), one gets that $|\omega| \leq\|\Delta\|$, whence $|\omega| \tau_{i} \leq\|\Delta\| \bar{\tau}<\pi / 2$. In these conditions, the terms $\cos \omega \tau_{i}$ appearing in (35a) are all positive. Note that not all the coefficients $\alpha_{i}$ can be zero, otherwise $\int_{G} u^{*} \cdot \Delta u=0$, which contradicts the fact that $u \in H^{1} \backslash\{0\}$. Therefore (35a) is impossible, and we are thus led to a contradiction. This proves that if $\tau_{i} \leq \bar{\tau}$ and (26) holds, then, the PdE (32) is globally asymptotically stable. 
We stress once more the robustness flavor of Theorem 5 , that requires just the knowledge of a common upper bound $\bar{\tau}$ on the (unknown) delays $\tau_{i}$. On the other hand, there may exist combinations of delays $\tau_{i}$ such that $\tau_{i} \geq \bar{\tau}$, for some $i \in \mathcal{I}$, but the $\operatorname{PdE}(32)$ remains asymptotically stable. An example is provided in Section 7.

The argument used in the proof of Theorem 4 does not seem to extend to the case of non-stationary delays. In this case, the next Theorem provides a sufficient stability condition.

Theorem 6 (Time-varying delays) The zero solution is a globally stable solution to the PdE (17) for all nonnegative, piecewise continuous delay $\tau_{i}(t)$ verifying $0 \leq \tau_{i}(t) \leq \bar{\tau}$, if

$$
\bar{\tau}<\frac{1}{\sum_{i, i^{\prime} \in \mathcal{I}}\left\|\Delta_{i} \Delta_{i^{\prime}}\right\|\left\|\Delta^{-1}\right\|} .
$$

Theorem 6 is a direct consequence of the following stronger result.

Lemma 2 The zero solution is a globally stable solution to the equation (17) for all piecewise continuous nonnegative delay $\tau_{i}(t)$ verifying

$$
\sup _{t \in[0,+\infty)}\left(\sum_{i, i^{\prime} \in \mathcal{I}} \tau_{i}(t)\left\|\Delta_{i} \Delta_{i^{\prime}}\right\|\right)<1 /\left\|\Delta^{-1}\right\|
$$

Proof: One may write

$$
\begin{aligned}
\dot{v}_{1} & =\sum_{i \in \mathcal{I}} \Delta_{i} v_{1}\left(x, t-\tau_{i}(t)\right)=\Delta v_{1}(x, t)+\sum_{i \in \mathcal{I}} \Delta_{i}\left(v_{1}\left(x, t-\tau_{i}(t)\right)-v_{1}(x, t)\right) \\
& =\Delta v_{1}(x, t)-\sum_{i \in \mathcal{I}} \Delta_{i} \int_{t-\tau_{i}(t)}^{t} \dot{v}_{1}(x, s) d s \\
& =\Delta v_{1}(x, t)-\sum_{i, i^{\prime} \in \mathcal{I}} \Delta_{i} \int_{t-\tau_{i}(t)}^{t} \Delta_{i^{\prime}} v_{1}\left(x, s-\tau_{i^{\prime}}(s)\right) d s
\end{aligned}
$$

Let $\rho$ be the constant

$$
\rho \stackrel{\text { def }}{=} \sup _{t \in[0,+\infty)}\left(\sum_{i, i^{\prime} \in \mathcal{I}} \tau_{i}(t)\left\|\Delta_{i} \Delta_{i^{\prime}}\right\|\right)\left\|\Delta^{-1}\right\|<1 .
$$

By assumption, there exists a real number $q$ in the non empty interval $(1,1 / \rho)$.

We apply now Razumikhin theorem, see e.g. [9, Theorem 4.2] or [12, Chapter 4]. For $V\left(v_{1}\right) \doteq \frac{1}{2}\left\|v_{1}\right\|_{L^{2}}$, assume that $V\left(v_{1}(\cdot, s)\right)<q V\left(v_{1}(\cdot, t)\right)$ for all $s \in$ $[t-2 \bar{\tau}, t]$. From the expression of $\dot{v}_{1}$ in (38), one deduces that, along the 
trajectories of (17), it holds

$$
\begin{aligned}
\frac{d\left[V\left(v_{1}\right)\right]}{d t} & \leq\left(-\left\|\Delta^{-1}\right\|^{-1}+q\left(\sum_{i, i^{\prime} \in \mathcal{I}} \tau_{i}(t)\left\|\Delta_{i} \Delta_{i^{\prime}}\right\|\right)\right)\left\|v_{1}\right\|_{L^{2}} \\
& \leq\left\|\Delta^{-1}\right\|^{-1}(-1+q \rho)\left\|v_{1}\right\|_{L^{2}} .
\end{aligned}
$$

Then, $\frac{d\left[V\left(v_{1}\right)\right]}{d t}<0$ and the thesis follows.

\begin{tabular}{|c|c|c|c|c|}
\hline $\bar{\tau}$ & Uni & rm delays & \multicolumn{2}{|c|}{ Non-uniform delays } \\
\hline Time-invariant delays & $\frac{\pi}{2\|\Delta\|}$ & Th. 3, (E) & $\frac{\pi}{2\|\Delta\|} \quad$ Th. 5 & (E) \\
\hline Time-varying delays & $\frac{3}{2\|\Delta\|}$ & Th. 4, (E) & $\frac{1}{\sum_{i, i^{\prime} \in \mathcal{I}}\left\|\Delta_{i} \Delta_{i^{\prime}}\right\|\left\|\Delta^{-1}\right\|}$ & Th. 6, (s) \\
\hline
\end{tabular}

The results in Theorems 3, 4, 5 and 6 are summarized in Table 1.

Table 1

Bounds on the worst-case stabilizing delay. E: exact, s: sufficient.

Remark 2 By comparison with (31), the bound (36) depends in a more involved manner upon the structure of the communication network. We also highlight that the bound (36) may be bounded from above by the simpler quantity $1 /(\operatorname{Tr} \Delta)^{2}\left\|\Delta^{-1}\right\|$, where $\operatorname{Tr} \Delta$ is the trace of the Laplacian on $H^{1}$.

For checking that the results of Theorems 4 and 6 are coherent, one can use the following inequalities

$$
\begin{aligned}
& \sum_{i, i^{\prime} \in \mathcal{I}}\left\|\Delta_{i} \Delta_{i^{\prime}}\right\|\left\|\Delta^{-1}\right\| \geq\left\|\sum_{i, i^{\prime} \in \mathcal{I}} \Delta_{i} \Delta_{i^{\prime}}\right\|\left\|\Delta^{-1}\right\|= \\
& =\left\|\left(\sum_{i \in \mathcal{I}} \Delta_{i}\right)^{2}\right\|\left\|\Delta^{-1}\right\|=\left\|\Delta^{2}\right\|\left\|\Delta^{-1}\right\| \geq\|\Delta\|,
\end{aligned}
$$

that imply $(36) \leq \frac{1}{\|\Delta\|} \leq \frac{3}{2\|\Delta\|}$. Also, we highlight the trade-off between stability with large delays on the one hand, and large decay-rate of the solutions on the other hand: the first one requires a small $\|\Delta\|$, whereas the second one requires a large $\left\|\Delta^{-1}\right\|^{-1} \leq\|\Delta\|$.

\section{A delay-independent condition for average consensus}

According to the standard terminology in time-delay systems, all the results presented in Sections 4 and 5 are "delay-dependent" in the sense that they 
guarantee average consensus when all the communication delays are upperbounded by a suitable value $\bar{\tau}$. Next, we show that if a single delay is zero, average consensus may be achieved irrespectively of the magnitude of all other delays. In this sense, we provide a "delay-independent" condition for average consensus. For two operators $A$ and $B$ from $L^{2}$ to $L^{2}$ the inequality $A>B$ on $H^{1}$ means that

$$
\forall u \in H^{1} \backslash\{0\},(u,(A-B) u)_{L^{2}}>0
$$

Theorem 7 Consider the PdE (32) and assume that $\tau_{i^{\prime}}=0$ for an index $i^{\prime} \in \mathcal{I}$. If

$$
\Delta_{i^{\prime}}<\sum_{i \in \mathcal{I} \backslash\left\{i^{\prime}\right\}} \Delta_{i} \text { on } H^{1}
$$

then, the zero solution is a globally exponentially stable solution to (32) for any $\tau_{i} \geq 0, i \in \mathcal{I} \backslash\left\{i^{\prime}\right\}$. Conversely, if the zero solution to system (32) with $\tau_{i^{\prime}}=0$ is globally asymptotically stable for any $\tau_{i} \geq 0, i \in \mathcal{I} \backslash\left\{i^{\prime}\right\}$, then

$$
\Delta_{i^{\prime}} \leq \sum_{i \in \mathcal{I} \backslash\left\{i^{\prime}\right\}} \Delta_{i} \quad \text { on } H^{1} .
$$

Proof: Assume first that (40) holds. Then, for any $z_{i} \in \mathbb{C}, i \in \mathcal{I} \backslash\left\{i^{\prime}\right\}$, such that $\left|z_{i}\right| \leq 1$, one has

$$
\left(\Delta_{i^{\prime}}+\sum_{i \in \mathcal{I} \backslash\left\{i^{\prime}\right\}} z_{i} \Delta_{i}\right)+\left(\Delta_{i^{\prime}}+\sum_{i \in \mathcal{I} \backslash\left\{i^{\prime}\right\}} z_{i} \Delta_{i}\right)^{*} \leq 2\left(\Delta_{i^{\prime}}-\sum_{i \in \mathcal{I} \backslash\left\{i^{\prime}\right\}} \Delta_{i}\right)<0 \text { on } H^{1}
$$

Consequently, $\Delta_{i^{\prime}}+\sum_{i \in \mathcal{I} \backslash\left\{i^{\prime}\right\}} z_{i} \Delta_{i}$ has only eigenvalues with strictly negative real part on $H^{1}$ for any $\left|z_{i}\right| \leq 1$. This implies that, for any $s \in \mathbb{C}$ with $\operatorname{Re} s \geq 0$, the following inequality holds on $H^{1}$ :

$$
\begin{aligned}
\left(s+s^{*}\right) I \geq 0>2\left(\Delta_{i^{\prime}}\right. & \left.+\sum_{i \in \mathcal{I} \backslash\left\{i^{\prime}\right\}} \Delta_{i}\right) \\
& \geq\left(\Delta_{i^{\prime}}+\sum_{i \in \mathcal{I} \backslash\left\{i^{\prime}\right\}} z_{i} \Delta_{i}\right)+\left(\Delta_{i^{\prime}}+\sum_{i \in \mathcal{I} \backslash\left\{i^{\prime}\right\}} z_{i} \Delta_{i}\right)^{*} .
\end{aligned}
$$

In particular, one can choose $z_{i}=e^{-s \tau_{i}}$ in (42), because $\left|e^{-s \tau_{i}}\right| \leq 1$ when Re $s \geq 0$ and $\tau_{i} \geq 0$. Thus,

$$
\left(s I-\Delta_{i^{\prime}}-\sum_{i \in \mathcal{I} \backslash\left\{i^{\prime}\right\}} e^{-s \tau_{i}} \Delta_{i}\right)+\left(s I-\Delta_{i^{\prime}}-\sum_{i \in \mathcal{I} \backslash\left\{i^{\prime}\right\}} e^{-s \tau_{i}} \Delta_{i}\right)^{*}>0
$$

for any $\tau_{i} \geq 0, i \in \mathcal{I} \backslash\left\{i^{\prime}\right\}$. As a consequence, all the roots of the characteristic equation $\operatorname{det}\left(s I-\Delta_{i^{*}}-\sum_{i \in \mathcal{I} \backslash\left\{i^{*}\right\}} e^{-s \tau_{i}} \Delta_{i}\right)=0$ of (32) have strictly negative 


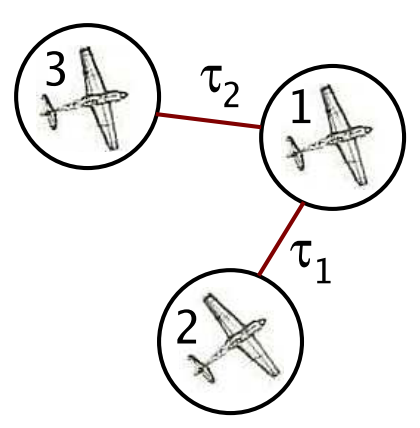

Fig. 2. The multi-agent system, with the communication delays, used in Section 7. real part. This yields the delay-independent asymptotic stability of (32).

Conversely, assume that (41) is not fulfilled. Then,

$$
\Delta_{i^{\prime}}-\sum_{i \in \mathcal{I} \backslash\left\{i^{\prime}\right\}} \Delta_{i}
$$

admits a real positive eigenvalue. In these conditions, system (32) is not delayindependently stable, see $[10,8]$.

\section{$7 \quad$ Examples}

We stress once more that the results in Sections 4 and 5 characterize robustness of average consensus, i.e. average consensus for any value of the delays less or equal to $\bar{\tau}$. In order to illustrate this concept, we consider the network of three agents whose communication graph $G$ is represented in Figure 2.

We assume that $v(x, t) \in \mathbb{R}^{2}$, that the weights $\omega(x, y)=1 \Leftrightarrow x \sim y$ are used and that the delays $\tau_{i}>0, i=1,2$ are constant in time. Moreover, the agents evolve according to the $\mathrm{PdE}$ (17) starting from the initial conditions

$$
\tilde{v}(1, t)=[2,2]^{\prime}, \quad \tilde{v}(2, t)=[2,-2]^{\prime}, \quad \tilde{v}(3, t)=[1,3]^{\prime}
$$

where $t \in\left[-\max \left\{\tau_{1}, \tau_{2}\right\}, 0\right]$. The average velocity at time $t=0$ is $\bar{v}=\left[\frac{5}{3}, 1\right]^{\prime}$. From Theorem 1, the eigenvalues of $\Delta$ on $H^{1}$ are the non null eigenvalues of $\mathcal{M}(\Delta)$ (up to their multiplicity). In our case, one gets $\|\Delta\|=3$, and the bound (26) is equal to $\pi / 6 \simeq 0.524$.

In the first experiment, we choose the delays $\tau_{1}=\tau_{2}=0.51$ that are slightly below $\bar{\tau}$. Then, Theorem 3 guarantees average consensus and such a result can be verified from Figure 3(a), where the evolution of $\|v(x, t)-\bar{v}\|, x \in\{1,2,3\}$ is represented. In the second experiment, we use $\tau_{1}=\tau_{2}=0.53$, so having $\tau_{1}=$ $\tau_{2}>\bar{\tau}$. As predicted by Theorem 3, the dynamics of $v_{1}$ becomes unstable and average consensus cannot be achieved. This can be clearly seen in Figure 3(b). Finally, we choose $\tau_{1}=0.1$ and $\tau_{2}=0.7$. In this case, $\tau_{2}$ violates the bound of 

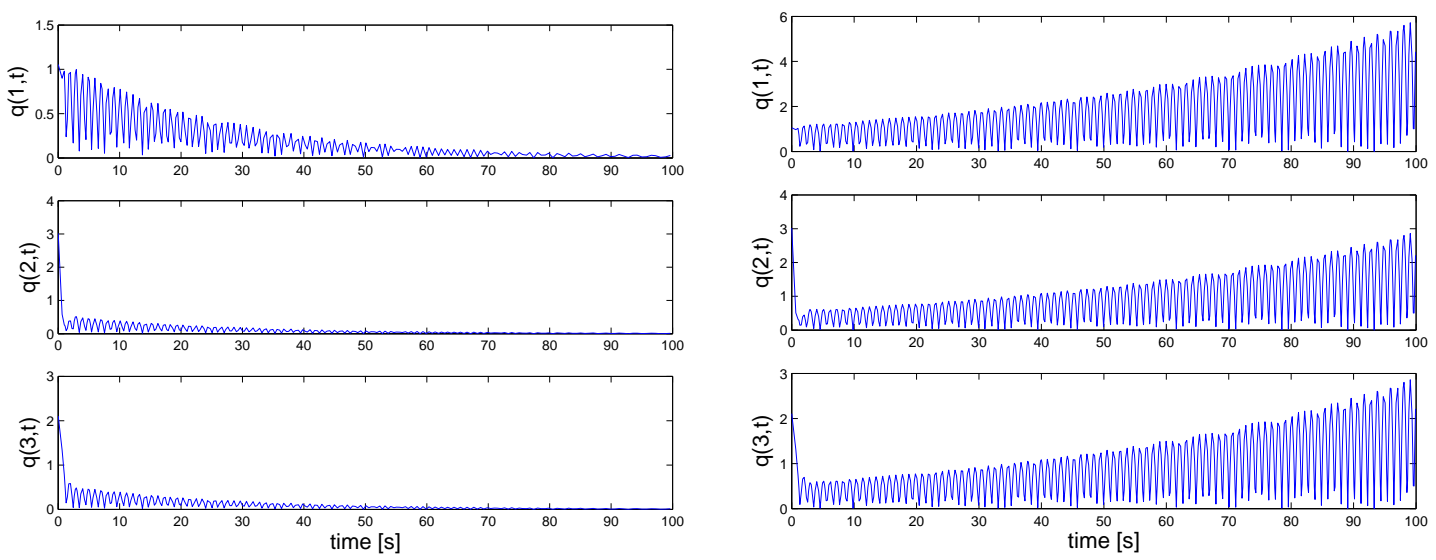

(a) $\tau_{1}=\tau_{2}=0.51$

(b) $\tau_{1}=\tau_{2}=0.53$

Fig. 3. Time evolution of $q(x, t)=\|v(x, t)-\bar{v}\|$ for the multi-agent system in Figure 2 .
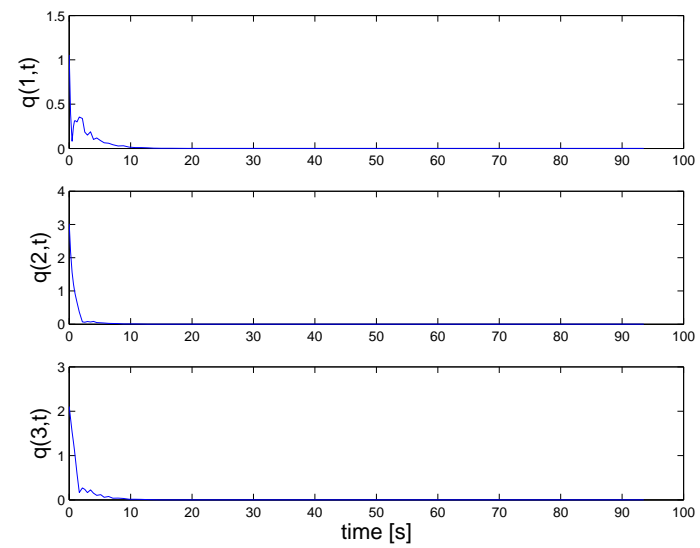

Fig. 4. Time evolution of $q(x, t)=\|v(x, t)-\bar{v}\|$ for the multi-agent system in Figure 2 , with $\tau_{1}=0.1$ and $\tau_{2}=0.7$.

Theorem 3. However, $\tau_{1}<\bar{\tau}$ and Theorem 3 cannot be used for checking the average consensus property. In this case, the achievement of average consensus can be verified by simulation, as shown in Figure 4 .

\subsection{Two extremal cases}

We now consider the case of complete and loop-shaped graphs with uniform weights. In Sections 7.1.1 and 7.1.2 it is shown that the bounds of Table 1 can be computed in closed-form as a function of $N$.

\subsubsection{Complete graph}

We consider a complete graph $G$ characterized by $\mathcal{E}=\mathcal{N} \times \mathcal{N}$ and $\omega(x, y)=$ $\delta>0$. We assume that a single delay $\tau_{x, y}$ is associated to each edge. Since 
delays are symmetric, then $\mathcal{D}=\left\{\tau_{x, y},(x, y) \in \mathcal{I}\right\}$ where $\mathcal{I}=\{(x, y) \in G \times G$ : $x<y\}$ and the cardinality of $r$ of $\mathcal{I}$, defined in (2), is $\frac{N(N-1)}{2}$. Note that the only difference with respect to $(2)$ is that delays are parametrized by two indexes. One also has that $G_{x, y}=\left(\mathcal{N}, T^{-1}\left(\tau_{x, y}\right)=\{(x, y),(y, x)\}\right.$ and, by direct computation,

$$
\left(\Delta_{x, y} f\right)(\xi)= \begin{cases}0 & \text { if } \xi \notin G_{x, y} \\ \delta(f(y)-f(x)) & \text { if } \xi=x \\ \delta(f(x)-f(y)) & \text { if } \xi=y\end{cases}
$$

The matrix representation of $\Delta_{x, y}$ is given by

$$
\mathcal{M}\left(\Delta_{x, y}\right)=-\delta e_{x, y} e_{x, y}^{T} \otimes I_{d}
$$

where $e_{x, y}$ is defined as the vector in $\mathbb{R}^{N}$ with zero entries, except the $x$-th one, equal to 1 , and the $y$-th one, equal to -1 . Note that, from (10), we have

$$
\Delta=\sum_{x<y} \Delta_{x, y}
$$

For computing the bound in (36), one needs to evaluate $\left\|\Delta_{x^{\prime}, y^{\prime}} \Delta_{x, y}\right\|$ for $x<y$ and $x^{\prime}<y^{\prime}$. Note that $\left(\Delta_{x^{\prime}, y^{\prime}} \Delta_{x, y} f\right)(\xi)$ is possibly non null only if the indexes $\left(x^{\prime}, y^{\prime}\right)$ and $(x, y)$ have both elements in common (i.e. $\left.\left(x^{\prime}, y^{\prime}\right)=(x, y)\right)$ or if they have just one element in common. The latter occours if and only if

$x<y, x^{\prime}<y^{\prime}$ and $\left(x=x^{\prime}, y \neq y^{\prime}\right.$ or $x \neq x^{\prime}, y=y^{\prime}$ or $x=y^{\prime}, y \neq x^{\prime}$ or $\left.x \neq y^{\prime}, y=x^{\prime}\right)$

The next lemma provides closed-form expression for the quantities appearing in Table 1.

Lemma 3 For a complete graph $G$ with uniform weights $\omega(x, y)=\delta>0$ it holds

$$
\begin{gathered}
\|\Delta\|=\left\|\Delta^{-1}\right\|^{-1}=N \delta \\
\left\|e_{x, y} e_{x, y}^{T} e_{x^{\prime}, y^{\prime}} e_{x^{\prime}, y^{\prime}}^{T}\right\|= \begin{cases}4 & \text { if }\left(x^{\prime}, y^{\prime}\right)=(x, y) \\
1 & \text { if }\left(x^{\prime}, y^{\prime}\right) \text { and }(x, y) \text { verify }(47), \\
0 & \text { if } x \neq x^{\prime} \text { and } y \neq y^{\prime},\end{cases} \\
\sum_{x<y, x^{\prime}<y^{\prime}}\left\|\Delta_{x^{\prime}, y^{\prime}} \Delta_{x, y}\right\|=\delta^{2} N^{2}(N-1) .
\end{gathered}
$$

Proof: In [3, pag. 269] it is shown that the non-zero eigenvalues of $\mathcal{M}(\Delta)$ are all equal to $-N$, when $\delta=1$. In view of Theorem 1 , these are the eigenvalues of $\Delta$ on $H^{1}$ and (48a) follows. A simple proof of the same fact is given next. From the graph completeness, one has $\Delta f(x)=-\delta N f(x)+\delta \int_{G} f(y) d y$. Then, $\Delta f=\lambda f, f \in H^{1}$ results in

$$
-\delta N f(x)+\delta \int_{G} f(y) d y=\lambda f(x)
$$


and since the average of $f$ is zero,

$$
-\delta N f(x)=\lambda f(x)
$$

so proving that the eigenvalues of $\Delta$ on $H^{1}$ are all equal to $-N \delta$.

Formula (48b) is obtained directly. When $\left(x^{\prime}, y^{\prime}\right)=(x, y)$ we have that $\left(e_{x, y} e_{x, y}^{T}\right)^{2}$ has all zero entries except the elements $(x, x),(y, y)$ (that are equal to 2$)$ and $(x, y),(y, x)$ (that are equal to -2$)$. The minimal eigenvalue of $\left(e_{x, y} e_{x, y}^{T}\right)^{2}$ is always -4 . When $\left(x^{\prime}, y^{\prime}\right)$ and $(x, y)$ verify $(47)$, one can show that up to a permutation of the agent indexes, which does not affect the results, it holds

$$
\left\|e_{x, y} e_{x, y}^{T} e_{x^{\prime}, y^{\prime}} e_{x^{\prime}, y^{\prime}}^{T}\right\|=\left\|\left(\begin{array}{ccc}
1 & -1 & 0 \\
-1 & 1 & 0 \\
0 & 0 & 0
\end{array}\right)\left(\begin{array}{ccc}
0 & 0 & 0 \\
0 & 1 & -1 \\
0 & -1 & 1
\end{array}\right)\right\|=\left\|\left(\begin{array}{ccc}
0 & 1 & -1 \\
0 & -1 & 1 \\
0 & 0 & 0
\end{array}\right)\right\|=1 .
$$

Finally, from (48b), one gets

$$
\sum_{x<y, x^{\prime}<y^{\prime}}\left\|e_{x, y} e_{x, y}^{T} e_{x^{\prime}, y^{\prime}} e_{x^{\prime}, y^{\prime}}^{T}\right\|=4 \sum_{x=x^{\prime}<y=y^{\prime}} 1+1 \sum_{\substack{(x, y),\left(x^{\prime}, y^{\prime}\right) \\ \text { verifying }(47)}} 1
$$

The first sum in the previous formula is equal to $\frac{N(N-1)}{2}$. As for the second sum, for a fixed pair $(x, y), 1 \leq x<y \leq N$ one can show that the number of pairs $\left(x^{\prime}, y^{\prime}\right)$ verifying $1 \leq x^{\prime}<y^{\prime} \leq N, x<y, x^{\prime}<y^{\prime}$ and

$$
\begin{aligned}
& x=x, y \neq y^{\prime} \text { is } N-x-1 \\
& x \neq x, y=y^{\prime} \text { is } y-2 \\
& x=y^{\prime}, y \neq x^{\prime} \text { is } x-1 \\
& x \neq y^{\prime}, y=x^{\prime} \text { is } N-y
\end{aligned}
$$

Then,

$$
\sum_{\substack{(x, y),\left(x^{\prime}, y^{\prime}\right) \\ \text { verifying }(47)}} 1=\frac{N(N-1)}{2}(N-x-1+y-2+x-1+N-y)=N(N-1)(N-2)
$$

and one finally gets

$$
\sum_{x<y, x^{\prime}<y^{\prime}}\left\|e_{x, y} e_{x, y}^{T} e_{x^{\prime}, y^{\prime}} e_{x^{\prime}, y^{\prime}}^{T}\right\|=\frac{N(N-1)}{2}+N(N-1)(N-2)=N^{2}(N-1)
$$


From (45), one has that $\mathcal{M}\left(\Delta_{x, y} \Delta_{x^{\prime}, y^{\prime}}\right)=\delta^{2} e_{x, y} e_{x, y}^{T} e_{x^{\prime}, y^{\prime}} e_{x^{\prime}, y^{\prime}}^{T}$ and formula (48c) follows.

\subsubsection{Loop-shaped graph}

We now analyze the case of a loop-shaped graph with uniform weights where each agent exchanges information only with two other ones. More precisely, the set of edges is given by $\mathcal{E}=\{(1,2),(2,3), \ldots,(N-1, N),(N, 1)\}$ and $\omega(x, y)=\delta>0$ if $(x, y) \in \mathcal{E}$. As in Section 7.1.1, we associate a different delay to each edge. Then, $\mathcal{D}=\left\{\tau_{x, y},(x, y) \in \mathcal{I}\right\}$, where $\mathcal{I}=\mathcal{E}$. The operators $\Delta_{x, y}$ are defined as in (44) and their matrix representation is given in (45). The matrix representation of $\Delta$ is given by

$$
\mathcal{M}(\Delta)=\delta\left(-2 I_{N}+P_{N}+P_{N}^{T}\right) \otimes I_{d}
$$

. where $P_{N} \in \mathbb{R}^{N \times N}$ is the permutation matrix

$$
P_{N}=\left(\begin{array}{ccccc}
0 & 0 & \cdots & 0 & 1 \\
1 & 0 & \cdots & 0 & 0 \\
0 & 1 & \cdots & 0 & 0 \\
\vdots & \vdots & \ddots & \vdots & \vdots \\
0 & 0 & \cdots & 1 & 0
\end{array}\right)
$$

The decomposition (10) becomes

$$
\Delta=-\delta \sum_{(x, y) \in \mathcal{I}} e_{x, y} e_{x, y}^{T} \otimes I_{d}
$$

where the definition of $e_{x, y}$, has been given in Section 7.1.1.

The counterpart of Lemma 3 is stated next.

Lemma 4 For a loop-shaped graph $G$ with uniform weights $\omega(x, y)=\delta>0$ it holds

$$
\begin{gathered}
\|\Delta\|=4 \sin ^{2}\left(\left\lfloor\frac{N}{2}\right\rfloor \frac{\pi}{N}\right), \quad\left\|(\Delta)^{-1}\right\|^{-1}=4 \delta \sin ^{2}\left(\frac{\pi}{N}\right) \\
\sum_{(x, y),\left(x^{\prime}, y^{\prime}\right) \in \mathcal{I}}\left\|\Delta_{x^{\prime}, y^{\prime}} \Delta_{x, y}\right\|=6 \delta^{2} N .
\end{gathered}
$$

Proof: As is well-known, $P_{N}^{T}=P_{N}^{-1}$, and the eigenvalues $\lambda_{P_{N}}$ of $P_{N}$ are all the $N$-th roots of the unit, i.e. $\lambda_{P_{N}}=\exp \left(\frac{2 \pi k}{N}\right), k=1, \ldots, N$. An eigenvalue $\lambda$ of $\mathcal{M}(\Delta)$ fulfills the equation

$$
\left(P_{N}+P_{N}^{-1}\right) \psi=\tilde{\lambda} \psi, \quad \tilde{\lambda}=\lambda+2
$$


from which $\tilde{\lambda}=\lambda_{P_{N}}+\lambda_{P_{N}}^{-1}=2 \cos \left(\frac{2 \pi k}{N}\right)$. Then, $\lambda=2 \cos \left(\frac{2 \pi k}{N}\right)-2=$ $-4 \sin ^{2} \frac{k \pi}{N}$. From Theorem 1 , a nonzero $\lambda$ is an eigenvalue of $\Delta$ on $H^{1}$. This allows to deduce the identities in (58a).

From identity (48b), it turns out that

$$
\sum_{(x, y) \in \mathcal{I},\left(x^{\prime}, y^{\prime}\right) \in \mathcal{I}}\left\|e_{x, y} e_{x, y}^{T} e_{x^{\prime}, y^{\prime}} e_{x^{\prime}, y^{\prime}}^{T}\right\|=4 \sum_{(x, y)=\left(x^{\prime} y^{\prime}\right) \in \mathcal{I}} 1+1 \sum_{\substack{(x, y),\left(x^{\prime}, y^{\prime}\right) \\ \text { verifying (47) }}} 1
$$

The first sum in (59) is equal to $N$. By direct calculation, the number of pairs $(x, y) \in \mathcal{I}$ that verify (47) for a given pair $\left(x^{\prime}, y^{\prime}\right) \in \mathcal{I}$ is 2 . Therefore, since the number of possible pairs $\left(x^{\prime}, y^{\prime}\right) \in \mathcal{I}$ is $N$, one has that the total number of pairs $\left(x^{\prime}, y^{\prime}\right)$ and $(x, y)$ that verify $(47)$ is $2 N$. Then, one obtains

$$
\sum_{(x, y) \in \mathcal{I},\left(x^{\prime}, y^{\prime}\right) \in \mathcal{I}}\left\|e_{x, y} e_{x, y}^{T} e_{x^{\prime}, y^{\prime}} e_{x^{\prime}, y^{\prime}}^{T}\right\|=4 N+2 N=6 N
$$

From (45), one has that $\mathcal{M}\left(\Delta_{x, y} \Delta_{x^{\prime}, y^{\prime}}\right)=\delta^{2} e_{x, y} e_{x, y}^{T} e_{x^{\prime}, y^{\prime}} e_{x^{\prime}, y^{\prime}}^{T}$. and formula (58b) follows.

The delay margins obtained by applying the results summarized in Table 1 are given now in Tables 2 and 3, for each of the two networks.

Table 2

\begin{tabular}{c||c|c|}
$\bar{\tau}$ & Uniform delays & Non-uniform delays \\
\hline Time-invariant delays & $\frac{\pi}{2 \delta N}$ & $\frac{\pi}{2 \delta N}$ \\
\hline Time-varying delays & $\frac{3}{2 \delta N}$ & $\frac{1}{\delta N(N-1)}$ \\
\hline
\end{tabular}

Complete graph: Bounds on the worst-case stabilizing delays

\section{Conclusions}

We provided convergence analysis of an average consensus protocol for undirected networks of dynamic agents having communication delays. We considered constant or time-varying delays, uniformly or non uniformly distributed in the network. Sufficient conditions (also necessary in most cases) for existence of average consensus under bounded, but otherwise unknown, communication delays, have been given. Simulations have been provided that demonstrate adequation with the bounds computed analytically. 
Table 3

\begin{tabular}{c||c|c|}
$\bar{\tau}$ & Uniform delays & Non-uniform delays \\
\hline Time-invariant delays & $\frac{\pi}{8 \delta \sin ^{2}\left(\left\lfloor\frac{N}{2}\right\rfloor \frac{\pi}{N}\right)}$ & $\frac{\pi}{8 \delta \sin ^{2}\left(\left\lfloor\frac{N}{2}\right\rfloor \frac{\pi}{N}\right)}$ \\
\hline Time-varying delays & $\frac{3}{8 \delta \sin ^{2}\left(\left\lfloor\frac{N}{2}\right\rfloor \frac{\pi}{N}\right)}$ & $\frac{2 \sin ^{2} \frac{\pi}{N}}{3 \delta N}$ \\
\hline
\end{tabular}

Loop-shaped graph: Bounds on the worst-case stabilizing delays.

\section{References}

[1] D. Angeli and P.-A. Bliman. Stability of leaderless muti-agent systems. extension of a result by moreau. Technical report, arXiv:math.OC/0411338, http://arxiv.org/, 2004.

[2] A. Bensoussan and J.-L. Menaldi. Difference equations on weighted graphs. Journal of Convex Analysis (Special issue in honor of Claude Lemaréchal), 12(1):13-44, 2005.

[3] B. Bollobs. Modern graph theory. Graduate texts in Mathematics. SpringerVerlag, 1998.

[4] G. Ferrari-Trecate, A. Buffa, and M. Gati. Analysis of coordination in multiple agents formations through partial difference equations. Technical report, N.5PV, Istituto di Matematica Applicata e Tecnologie Informatiche, C.N.R., Pavia, Italy.

http://www-rocq.inria.fr/who/Giancarlo.Ferrari-Trecate/FTBG04.html 2004.

[5] E. Franco, T. Parisini, and M.M. Polycarpou. Cooperative control of discretetime agents with delayed information excange: a receding-horizon approach. In Proceedings of the $44^{\text {nd }}$ IEEE Conference on Decision and Control, pages 4727-4279, 2004.

[6] R. Grone and R. Merris. The Laplacian spectrum of a graph. II. SIAM J. Discrete Math., 7(2):221-229, 1994.

[7] R. Grone, R. Merris, and V.S. Sunder. The Laplacian spectrum of a graph. SIAM J. Matrix Anal. Appl., 11(2):218-238, 1990.

[8] J.K. Hale, E.F. Infante, and F.S.P. Tsen. Stability in linear delay equations. J. Math. Anal. Appl., 115:533-555, 1985.

[9] J.K. Hale and S.M. Verduyn Lunel. Introduction to Functional Differential Equations. Applied Mathematical Sciences 99. Springer-Verlag, New York, 1993. 
[10] D. Hertz, E.I. Jury, and E. Zeheb. Stability independent and dependent of delay for delay differential systems. J. Franklin Institute, 318(3):143-150, 1984.

[11] A. Jadbabaie, J. Lin, and A. S. Morse. Coordination of groups of mobile autonomous agents using nearest neighbor rules. IEEE Trans. on Automatic Control, 48(6):988 - 1001, 2003.

[12] V. Kolmanovskii and A. Myshkis. Introduction to the Theory and Applications of Functional Differential Equations. Kluwer Academic Publishers, Dordrecht Boston London, 1999.

[13] R. Merris. Laplacian matrices of graphs: a survey. Linear Algebra Appl., 197:143-176, 1994.

[14] L. Moreau. Stability of continuous-time distributed consensus algorithms. Technical report, arXiv:math.OC/0306426, http://arxiv.org/, 2004.

[15] A.D. Myškis. On the solutions of linear homogeneous differential equations of the first order and stable type with retarded arguments (in russian). Matematicheski Sbornik, 28(70):641-658, 1951.

[16] R. Olfati-Saber and R. Murray. Consensus problems in networks of agents with switching topology and time-delays. IEEE Trans on Autom. Control, 49(9):101$115,2004$.

[17] R. Olfati Saber and R. Murray. Agreement problems in networks with directed graphs and switching topology. In Proceedings of the $42^{\text {nd }}$ IEEE Conference on Decision and Control, pages 4126-4132, 2003.

[18] H. Tanner, A. Jadbabaie, and G.J. Pappas. Stable flocking of mobile agents, part I : Fixed topology. In Proceedings of the $42^{\text {nd }}$ IEEE Conference on Decision and Control, pages 2010-2015, 2003.

[19] H. Tanner, A. Jadbabaie, and G.J. Pappas. Stable flocking of mobile agents, part II : Dynamic topology. In Proceedings of the $42^{\text {nd }}$ IEEE Conference on Decision and Control, pages 2016-2021, 2003.

[20] J.A. Yorke. Asymptotic stability for one dimensional differential-delay equations. Journal of Differential equations, 7:189-202, 1970. 